\title{
Note
}

\section{Total Synthesis of Thailandepsin B, a Potent HDAC Inhibitor Isolated from a Microorganism}

\author{
Koichi Narita and Tadashi Katoh* \\ Laboratory of Synthetic and Medicinal Chemistry, Faculty of Pharmaceutical Sciences, Tohoku \\ Medical and Pharmaceutical University; 4-4-1 Komatsushima, Aoba-ku, Sendai 981-8558, Japan. \\ Received January 20, 2016; accepted February 14, 2016
}

\begin{abstract}
Thailandepsin B, a bicyclic depsipeptide histone deacetylase inhibitor, was efficiently synthesized in $\mathbf{5 1 \%}$ overall yield in eight steps, starting from commercially available D-norleucine methyl ester and known $(S, E)$-3-(4-methoxybenzyloxy)-7-(tritylthio)hept-4-enoic acid. The method features a convergent approach in which the corresponding seco-acid, a key precursor in macrolactonization, is directly assembled through the condensation of a D-allo-isoleucine-D-cysteine-containing segment with a D-norleucine-containing segment.
\end{abstract} thesis

Key words thailandepsin B; histone deacetylase inhibitor; natural product; bicyclic depsipeptide; total syn-

In 2011, Cheng and colleagues reported the isolation and structural elucidation of the two bicyclic depsipeptide histone deacetylase (HDAC) inhibitors, thailandepsins A (1) and B (2) ${ }^{1)}$ (Chart 1). These compounds were identified through the systematic overexpression of transcription factors associated with natural product gene clusters encoded within Burkholdenia thailandensis E264. ${ }^{1)}$ Almost simultaneously, Brady and colleagues independently reported the identification of the two bicyclic depsipeptide HDAC inhibitors, burkholdacs A (3) and B (1), using the same bacterial strain and gene clusters but a different technical approach. ${ }^{2)}$ Coincidentally, burkholdac B was identical to thailandepsin $\mathrm{A}^{1,2}$ Thailandepsins $\mathrm{A}$ and $\mathrm{B}$ exhibit comparable HDAC inhibitory activity to that of FK228 (4; romidepsin), ${ }^{1)}$ which is an anticancer agent that has been approved by the U.S. Food and Drug Administration (FDA) for the treatment of cutaneous T-cell lymphoma. ${ }^{3,4)}$ Note that isozyme selectivity of thailandepsins is superior to that of $\mathbf{4}^{1 \text {; }}$; isozyme selectivity is critically important for developing new HDAC inhibitors as anticancer agents with reduced undesirable side effects. ${ }^{4,5}$ Thailandepsins, therefore, can be considered as intriguing and valuable targets for total synthesis. To date, the total synthesis of thailandepsin A (1; burkholdac B) has been reported by Ganesan and colleagues ${ }^{6}$ ) and our group. ${ }^{7)}$ However, the total synthesis of thailandepsin B (2) has only been reported once in the patent literature. ${ }^{8)}$

During our efforts synthesizing and biologically evaluating bicyclic depsipeptide HDAC inhibitors including burkholdacs A (3) ${ }^{7)}$ B (1, thailandepsin A) ${ }^{7)}$ FK228 (4, romidepsin) ${ }^{9)}$ and spiruchostatins A (5), ${ }^{9,10)} \mathrm{B}(\mathbf{6}),{ }^{9,11)} \mathrm{C}(\mathbf{7})^{12)}$ and D (8), ${ }^{12)}$ we became interested in the synthesis of thailandepsin B (2) and its analogues with the aim of identifying novel mechanism-based anticancer agents. In this study, we describe our total synthesis of 2 using a synthetic strategy developed previously in our laboratory., ${ }^{7,-12)}$

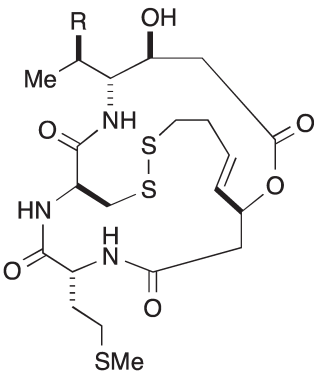

1 : thailandepsin $A(R=E t)$ ( burkholdac $B$ )

3 : burkholdac $A(R=M e)$

4 : FK228 ( romidepsin )

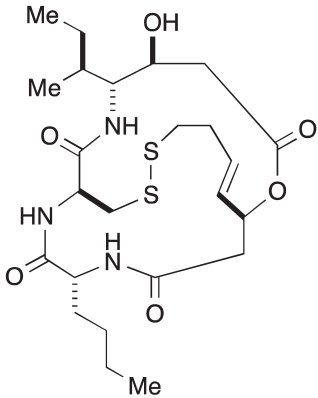

2 : thailandepsin $B$

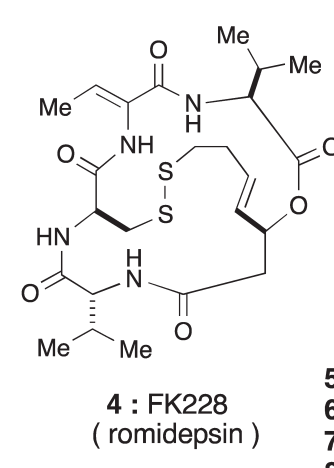

$i$-Pr=isopropyl, $s$-Bu=sec-butyl, and $i$ - $\mathrm{Bu}=$ isobutyl.

Chart 1. Structures of Thailandepsins A (1, Burkholdac B), B (2), Burkholdac A (3), FK228 (4; Romidepsin), and Spiruchostatins A-D (5-8)

\section{Results and Discussion}

Retrosynthetic analysis of thailandepsin B (2) is illustrated in Chart 2. We envisioned that target molecule $\mathbf{2}$ could be synthesized via the macrolactonization of the corresponding seco-acid 9 followed by internal disulfide bond formation. The 


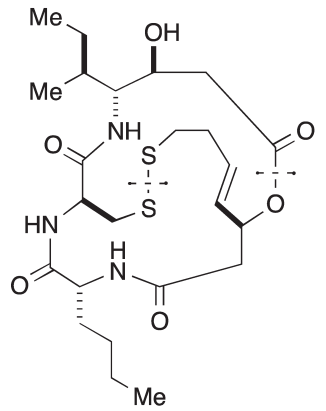

2 : thailandepsin B

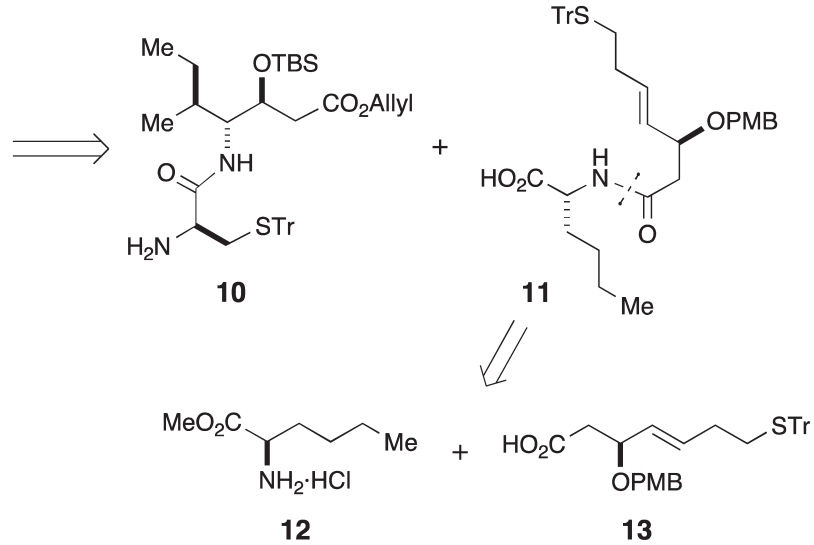

TBS=tert-butyldimethylsilyl; Tr=triphenylmethyl; PMB=4-methoxybenzyl

Chart 2. Retrosynthetic Analysis of Thailandepsin B (2)

key macrolactonization precursor 9 could be prepared through the direct condensation of the D-allo-isoleucine- and D-cysteine-containing segment $\mathbf{1 0}$ with the D-norleucine-containing segment 11. Segment 11 could be formed by the condensation of commercially available D-norleucine methyl ester hydrochloride (12) with the known carboxylic acid 13 (previously prepared from L-malic acid in our laboratory ${ }^{7,9-12)}$ ). Segment 10 was obtained as previously described in our total synthesis of 1 and $\mathbf{6}^{7,9)}$

We initially pursued the synthesis of the key segment $\mathbf{1 1}$, which is the coupling partner of $\mathbf{1 0}$, as shown in Chart 3. The condensation of 12 with $13^{7,9-12)}$ afforded the desired coupling product 14 in excellent yield (98\%). Subsequent saponification of the methyl ester moiety in $\mathbf{1 4}$ furnished the requisite segment 11 in $90 \%$ yield.

After obtaining the key segment 11, we directed our attention to the synthesis of the target thailandepsin B (2), as shown in Chart 4. The crucial condensation of $\mathbf{1 0}$ with $\mathbf{1 1}$ proceeded well under the mild conditions [ $O$-(7-azabenzotriazol-1-yl)$N, N, N^{\prime}, N^{\prime}$-tetramethyluronium hexafluorophosphate (HATU) (1.3 equiv), 1-hydroxy-7-azabenzotriazole (HOAt) (1.3 equiv), $i$ - $\mathrm{Pr}_{2} \mathrm{NEt}$ (2.6 equiv), $\mathrm{CH}_{2} \mathrm{Cl}_{2},-30^{\circ} \mathrm{C}, 5 \mathrm{~h}$ ] explored in our previous studies. ${ }^{7,9-12)}$ The desired condensation product $\mathbf{1 5}$ was obtained in $90 \%$ yield without appreciable epimerization at the $\mathrm{C} 2$ stereogenic center (D-norleucine part in 15). In this condensation reaction, the use of a combination of HATU and HOAt at low temperature was critical to effectively suppress unfavorable epimerization event. ${ }^{9)}$ The condensation product 15 was then converted to the requisite seco-acid 9 with an overall yield of $82 \%$, using the alcohol 16 by successive removal of both the 4-methoxybenzyl (PMB) and allyl-protecting groups. The subsequent macrolactonization of 9 was

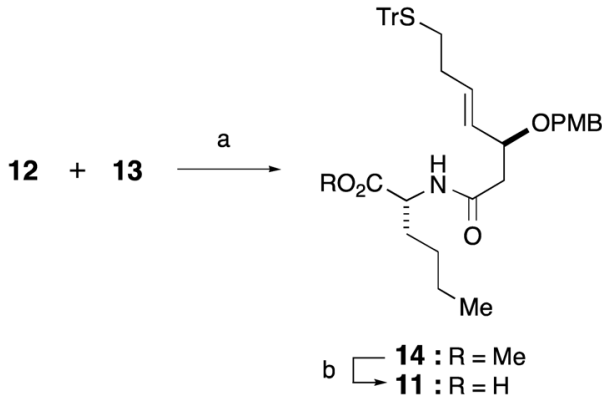

a) PyBOP, $i$-Pr ${ }_{2} \mathrm{NEt}, \mathrm{MeCN}, \mathrm{rt}, 98 \%$; b) $\mathrm{LiOH}, \mathrm{MeOH}, \mathrm{rt}, 90 \%$. PyBOP= (benzotriazol-1-yloxy)tripyrrolidinophosphonium hexafluorophosphate.

Chart 3. Synthesis of Segment 14

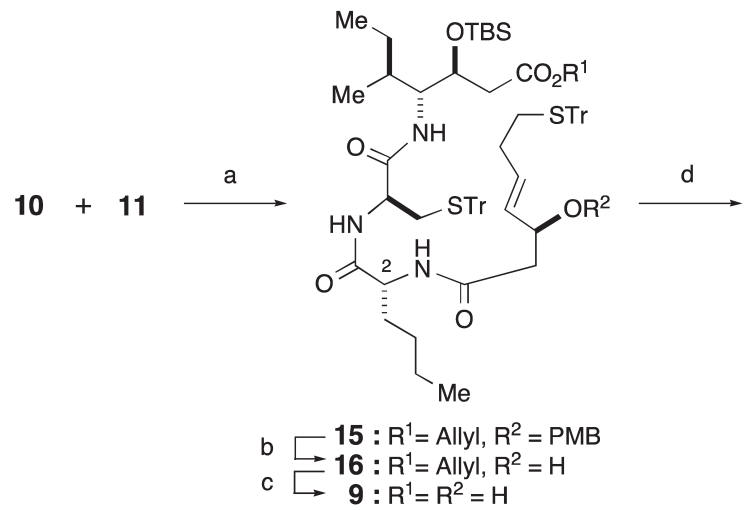

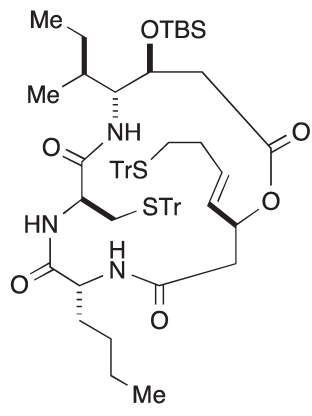

17

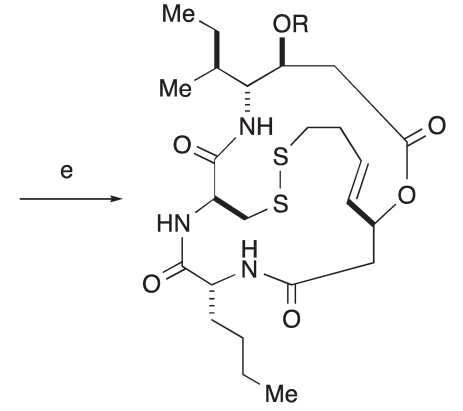

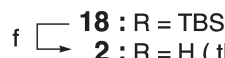

(a) HATU, HOAt, $i$ - $\mathrm{Pr}_{2} \mathrm{NEt}, \mathrm{CH}_{2} \mathrm{Cl}_{2},-30^{\circ} \mathrm{C}, 90 \%$; (b) $\mathrm{DDQ}, \mathrm{CH}_{2} \mathrm{Cl}_{2} / \mathrm{H}_{2} \mathrm{O}$, rt, $89 \%$; c) $\mathrm{Pd}\left(\mathrm{PPh}_{3}\right)_{4}$, morpholine, THF, rt, 92\%; (d) MNBA, DMAP, $\mathrm{CH}_{2} \mathrm{Cl}_{2}$, rt, $85 \%$; (e) $\mathrm{I}_{2}, \mathrm{CH}_{2} \mathrm{Cl}_{2}-\mathrm{MeOH}, \mathrm{rt}, 95 \%$; (f) $\mathrm{HF}$-pyridine, pyridine, rt, $90 \%$. HATU $=O$-(7-azabenzotriazol-1-yl)- $N, N, N^{\prime}, N^{\prime}$-tetramethyluronium hexafluorophosphate; HOAt=1-hydroxy-7-azabenzotriazole; $\mathrm{DDQ}=2,3$-dichloro-5,6-dicyano-1,4 benzoquinone; MNBA=2-methyl-6-nitrobenzoic anhydride; DMAP=4-dimethylaminopyridine.

Chart 4. Synthesis of Thailandepsin B (2)

achieved by employing the Shiina method ${ }^{13-17)}$ [2-methyl-6-nitrobenzoic anhydride (MNBA) (1.3 equiv), 4-dimethylaminopyridine (DMAP) (3.0 equiv), $\mathrm{CH}_{2} \mathrm{Cl}_{2}(1.0 \mathrm{~mm}), \mathrm{rt}, 12 \mathrm{~h}$ ], which resulted in the desired cyclization product $\mathbf{1 7}$ in $85 \%$ yield. Finally, simultaneous $S$-Tr deprotection and internal disulfide bond formation in 17 (95\%), followed by the deprotection of the TBS group in the resulting disulfide 18 (90\%) furnished thailandepsin B (2) with $[\alpha]_{\mathrm{D}}^{25}-28.4(c=1.0, \mathrm{MeCN})\left\{\right.$ lit. ${ }^{1)}[\alpha]_{\mathrm{D}}^{25}$ $-22.8(c=1.0, \mathrm{MeCN})\}$. The spectroscopic properties (infrared (IR), ${ }^{1} \mathrm{H}$ - and ${ }^{13} \mathrm{C}-\mathrm{NMR}$, and MS) of the synthetic sample 2 were identical to those reported for natural $2 .{ }^{1)}$ 


\section{Conclusion}

We have achieved the total synthesis of thailandepsin B (2) with $51 \%$ overall yield in eight steps, starting from commercially available D-norleucine methyl ester hydrochloride (12) and the known carboxylic acid 13. The method is in highly convergent manner and features the condensation of a D-allo-isoleucine-D-cysteine-containing segment $\mathbf{1 0}$ with a D-norleucine-containing segment $\mathbf{1 1}$ to directly construct the requisite peptide framework $\mathbf{1 5}(\mathbf{1 0}+\mathbf{1 1} \rightarrow \mathbf{1 5}$, Chart 4). The present study should be useful for producing thailandepsin analogues that possess various alkyl side chains. Further investigations concerning the synthesis and biological evaluation of thailandepsin analogues are currently under way and will be reported in due course.

\section{Experimental}

General Techniques All reactions involving air- and moisture-sensitive reagents were carried out using oven dried glassware and standard syringe-septum cap techniques. Routine monitorings of reaction were carried out using glasssupported Merck silica gel 60 F254 TLC plates. Flash column chromatography was performed on Kanto Chemical Silica Gel $60 \mathrm{~N}$ (spherical, neutral $40-50 \mathrm{~nm}$ ) with the solvents indicated.

All solvents and reagents were used as supplied with following exceptions. Tetrahydrofuran (THF) was freshly distilled from $\mathrm{Na}$ metal/benzophenone under argon. $\mathrm{CH}_{2} \mathrm{Cl}_{2}$, $\mathrm{MeCN}$, pyridine and $i$ - $\mathrm{Pr}_{2} \mathrm{NEt}$ were distilled from calcium hydride under argon.

Measurements of optical rotations were performed with a Anton Paar MCP-100 automatic digital polarimeter. ${ }^{1} \mathrm{H}$ and ${ }^{13} \mathrm{C}-\mathrm{NMR}$ spectra were measured with a JEOL AL-400 $(400 \mathrm{MHz})$ and JEOL LA-600 $(600 \mathrm{MHz})$ spectrometers. Chemical shifts were expressed in ppm using $\mathrm{Me}_{4} \mathrm{Si}(\delta=0)$ as an internal standard. The following abbreviations are used: singlet (s), doublet (d), triplet (t), quartet (q), multiplet (m) and broad (br). IR spectral measurements were carried out with a JASCO FT/IR-4100 spectrometer. Low- and high-resolution mass spectra (LR-HR-MS) were measured on a JEOL JMS-700 high resolution mass spectrometer.

(R)-Methyl 2-[(S,E)-3-(4-Methoxybenzyloxy)-7-(tritylthio)hept-4-enamido]hexanoate (14) $i$ - $\operatorname{Pr}_{2} \mathrm{NEt}(0.33 \mathrm{~mL}, 1.9 \mathrm{mmol})$ was added dropwise to a stirred solution of $\mathrm{D}$-norleucine methyl ester hydrochloride (12) $(88.0 \mathrm{mg}, 0.48 \mathrm{mmol})^{18)}$ and $(S, E)$-3-(4-methoxybenzyloxy)-7-(tritylthio)hept-4-enoic acid (13) ${ }^{7,9-12)}$ (174 mg, $\left.0.32 \mathrm{mmol}\right)$ in $\mathrm{MeCN}(6.5 \mathrm{~mL})$ containing (benzotriazol-1-yloxy)tripyrrolidinophosphonium hexafluorophosphate (PyBOP) $(252 \mathrm{mg}, 0.48 \mathrm{mmol})$ at $0^{\circ} \mathrm{C}$ under argon, and stirring was continued for $2 \mathrm{~h}$ at room temperature. The reaction mixture was diluted with EtOAc $(30 \mathrm{~mL})$, and the organic layer was washed successively with $10 \%$ aqueous $\mathrm{HCl}(2 \times 10 \mathrm{~mL})$, saturated aqueous $\mathrm{NaHCO}_{3}(2 \times 10 \mathrm{~mL})$ and brine $(2 \times 10 \mathrm{~mL})$, then dried over $\mathrm{Na}_{2} \mathrm{SO}_{4}$. Concentration of the solvent in vacuo afforded a residue, which was purified by column chromatography (hexane-EtOAc $2: 1)$ to give $14(211 \mathrm{mg}$, $98 \%)$ as a colorless viscous oil. $[\alpha]_{\mathrm{D}}^{25}-6.5\left(c=1.0, \mathrm{CHCl}_{3}\right)$; IR (neat) $\mathrm{cm}^{-1}: 3309,3057,2953,2929,2860,1743,1652$, 1613, 1514, 1443, 1362, 1248, 1080, 821, 744, 700; ${ }^{1} \mathrm{H}-\mathrm{NMR}$ $\left(400 \mathrm{MHz}, \mathrm{CDCl}_{3}\right) \delta: 0.83(3 \mathrm{H}, \mathrm{t}, J=7.1 \mathrm{~Hz}), 1.14-1.28(4 \mathrm{H}$, $\mathrm{m}), 1.53-1.60(1 \mathrm{H}, \mathrm{m}), 1.70-1.77(1 \mathrm{H}, \mathrm{m}), 2.04-2.25(4 \mathrm{H}, \mathrm{m})$, $2.38(1 \mathrm{H}, \mathrm{dd}, J=15.4,3.2 \mathrm{~Hz}), 2.51(1 \mathrm{H}, \mathrm{dd}, J=15.4,8.8 \mathrm{~Hz})$, $3.71(3 \mathrm{H}, \mathrm{s}), 3.78(3 \mathrm{H}, \mathrm{s}), 4.10(1 \mathrm{H}, \mathrm{td}, J=8.3,3.4 \mathrm{~Hz}), 4.31(1 \mathrm{H}$, d, $J=10.7 \mathrm{~Hz}), 4.51(1 \mathrm{H}, \mathrm{d}, J=10.7 \mathrm{~Hz}), 4.55(1 \mathrm{H}, \mathrm{td}, J=7.7$, $5.2 \mathrm{~Hz}), 5.32(1 \mathrm{H}, \mathrm{dd}, J=15.6,8.3 \mathrm{~Hz}), 5.59(1 \mathrm{H}, \mathrm{dt}, J=15.1$, $6.6 \mathrm{~Hz}), 6.81-6.84(2 \mathrm{H}, \mathrm{m}), 6.95(1 \mathrm{H}, \mathrm{d}, J=8.3 \mathrm{~Hz}), 7.19-7.29$ $(11 \mathrm{H}, \mathrm{m}), 7.41-7.45(6 \mathrm{H}, \mathrm{m}) ;{ }^{13} \mathrm{C}-\mathrm{NMR}\left(100 \mathrm{MHz}, \mathrm{CDCl}_{3}\right) \delta$ : $13.8,22.2,27.4,31.2,31.4,32.0,42.7,52.0,52.1,55.2,66.6$, 70.1, 76.8, 113.8 (2C), 126.6 (3C), 127.9 (6C), 129.5 (6C), 129.7 (2C), 130.0, 130.3, 132.9, 144.9 (3C), 159.2, 170.5, 173.0; HRFAB-MS $m / z$ : 688.3087 (Calcd for $\mathrm{C}_{41} \mathrm{H}_{47} \mathrm{NO}_{5} \mathrm{SNa}[\mathrm{M}+\mathrm{Na}]^{+}$: 688.3073).

(R)-2-[(S,E)-3-(4-Methoxybenzyloxy)-7-(tritylthio)hept-4-enamido]hexanoic Acid (11) One mol LiOH (1.30 mL, $1.3 \mathrm{mmol}$ ) was added dropwise to a stirred solution of $\mathbf{1 4}$ $(211 \mathrm{mg}, 0.32 \mathrm{mmol})$ in $\mathrm{MeOH}(6.3 \mathrm{~mL})$ at room temperature. After $3 \mathrm{~h}, 10 \%$ aqueous $\mathrm{HCl}$ was added to the mixture at $0^{\circ} \mathrm{C}$ until $\mathrm{pH}$ was 6 . The resulting mixture was extracted with EtOAc $(3 \times 30 \mathrm{~mL})$, and the combined extracts were washed with saturated aqueous $\mathrm{NaHCO}_{3}(2 \times 20 \mathrm{~mL})$ and brine $(2 \times 20 \mathrm{~mL})$, then dried over $\mathrm{Na}_{2} \mathrm{SO}_{4}$. Concentration of the solvent in vacuo afforded a residue, which was purified by column chromatography $\left(\mathrm{CHCl}_{3}-\mathrm{MeOH} 15: 1\right)$ to give $11(186 \mathrm{mg}, 90 \%)$ as a colorless amorphous solid. $[\alpha]_{\mathrm{D}}^{25}-2.4$ $\left(c=1.0, \mathrm{CHCl}_{3}\right)$; IR (neat) $\mathrm{cm}^{-1}: 3340,3057,2955,2930,2862$, 1732, 1614, 1514, 1449, 1443, 1248, 1079, 1034, 974, 822, 745, 701 ; ${ }^{1} \mathrm{H}-\mathrm{NMR}\left(400 \mathrm{MHz}, \mathrm{CDCl}_{3}\right) \delta: 0.82(3 \mathrm{H}, \mathrm{t}, J=6.8 \mathrm{~Hz})$, $1.21-1.27(4 \mathrm{H}, \mathrm{m}), 1.53-1.62(1 \mathrm{H}, \mathrm{m}), 1.75-1.84(1 \mathrm{H}, \mathrm{m})$, 2.04-2.24 (4H, m), $2.41(1 \mathrm{H}, \mathrm{dd}, J=15.4,3.2 \mathrm{~Hz}), 2.52(1 \mathrm{H}, \mathrm{dd}$, $J=15.1,8.8 \mathrm{~Hz}), 3.76(3 \mathrm{H}, \mathrm{s}), 4.10(1 \mathrm{H}, \mathrm{td}, J=8.2,3.4 \mathrm{~Hz}), 4.27$ $(1 \mathrm{H}, \mathrm{d}, J=10.7 \mathrm{~Hz}), 4.46-4.50(2 \mathrm{H}, \mathrm{m}), 5.30(1 \mathrm{H}, \mathrm{dd}, J=15.4$, $8.0 \mathrm{~Hz}), 5.58(1 \mathrm{H}, \mathrm{dt}, J=15.1,6.8 \mathrm{~Hz}), 6.79-6.83(2 \mathrm{H}, \mathrm{m}), 7.12$ $(1 \mathrm{H}, \mathrm{d}, J=7.3 \mathrm{~Hz}), 7.18-7.29(11 \mathrm{H}, \mathrm{m}), 7.39-7.42(6 \mathrm{H}, \mathrm{m})$; ${ }^{13} \mathrm{C}-\mathrm{NMR}\left(100 \mathrm{MHz}, \mathrm{CDCl}_{3}\right) \delta: 13.8,22.2,27.4,31.2,31.37$, 31.40, 42.4, 52.4, 55.2, 66.6, 70.1, 76.6, 113.9 (2C), 126.6 (3C), 127.9 (6C), 129.5 (6C), 129.7, 129.8 (2C), 130.1, 133.1, 144.9 (3C), 159.3, 171.6, 175.8; HR-FAB-MS m/z: 652.3094 (Calcd for $\mathrm{C}_{40} \mathrm{H}_{46} \mathrm{NO}_{5} \mathrm{~S}[\mathrm{M}+\mathrm{H}]^{+}$652.3097).

$(7 S, 11 R, 14 S, 17 R, 18 S, E)$-Allyl 17-[(S)-sec-Butyl]-11-butyl-18(tert-butyldimethylsilyloxy)-7-(4-methoxybenzyloxy)-9,12,15trioxo-1,1,1-triphenyl-14-(tritylthiomethyl)-2-thia-10,13,16triazaicos-5-en-20-oate (15) $\quad i$ - $\mathrm{Pr}_{2} \mathrm{NEt}(0.12 \mathrm{~mL}, 0.72 \mathrm{mmol})$ was added dropwise to a stirred solution of $(3 S, 4 R, 5 S)$ allyl-4-[(S)-2-amino-3-(tritylthio)propanamido]-3-(tertbutyldimethylsilyloxy)-5-methylheptanoate $(\mathbf{1 0})^{7,9)} \quad(187 \mathrm{mg}$, $0.28 \mathrm{mmol})$ and $11(186 \mathrm{mg}, 0.28 \mathrm{mmol})$ in $\mathrm{CH}_{2} \mathrm{Cl}_{2}(5.6 \mathrm{~mL})$ containing HATU $(137 \mathrm{mg}, 0.36 \mathrm{mmol})$ and HOAt $(49.1 \mathrm{mg}$, $0.36 \mathrm{mmol}$ ) at $-30^{\circ} \mathrm{C}$ under argon. After $5 \mathrm{~h}$, the reaction mixture was diluted with $\mathrm{CHCl}_{3}(50 \mathrm{~mL})$. The organic layer was washed successively with $10 \%$ aqueous $\mathrm{HCl}(2 \times 20 \mathrm{~mL})$, saturated aqueous $\mathrm{NaHCO}_{3}(2 \times 20 \mathrm{~mL})$ and brine $(2 \times 20 \mathrm{~mL})$, then dried over $\mathrm{MgSO}_{4}$. Concentration of the solvent in vacuo afforded a residue, which was purified by column chromatography (hexane/EtOAc $1: 1)$ to give $15(328 \mathrm{mg}, 90 \%)$ as a colorless viscous liquid. $[\alpha]_{\mathrm{D}}^{25}+13.1\left(c=1.0, \mathrm{CHCl}_{3}\right)$; IR (neat) $\mathrm{cm}^{-1}: 3285,3060,2956,2930,2857,1734,1637,1541,1515$, 1490, 1458, 1444, 1387, 1249, 1173, 1085, 1036, 938, 830, 744, 700; ${ }^{1} \mathrm{H}-\mathrm{NMR}\left(400 \mathrm{MHz}, \mathrm{CDCl}_{3}\right) \delta: 0.00(3 \mathrm{H}, \mathrm{s}), 0.07(3 \mathrm{H}$, s), $0.77-0.81(6 \mathrm{H}, \mathrm{m}),, 0.83-0.88(12 \mathrm{H}, \mathrm{m}), 1.05-1.28(6 \mathrm{H}$, m), $1.36-1.41(1 \mathrm{H}, \mathrm{m}), 1.64-1.69(1 \mathrm{H}, \mathrm{m}), 1.75-1.81(1 \mathrm{H}, \mathrm{m})$, 2.07-2.17 (2H, m), 2.20-2.25 (2H, m), 2.34 (1H, dd, $J=15.6$, $3.4 \mathrm{~Hz}), 2.40-2.58(4 \mathrm{H}, \mathrm{m}), 2.77(1 \mathrm{H}, \mathrm{dd}, J=12.7,7.8 \mathrm{~Hz})$, $3.77(3 \mathrm{H}, \mathrm{s}), 3.91-3.96(2 \mathrm{H}, \mathrm{m}), 4.01(1 \mathrm{H}, \mathrm{td}, J=8.0,3.3 \mathrm{~Hz})$, $4.10-4.20$ (3H, m), 4.38 (1H, d, $J=10.7 \mathrm{~Hz}), 4.44-4.55$ (2H, m), 
$5.17-5.31(3 \mathrm{H}, \mathrm{m}), 5.45(1 \mathrm{H}, \mathrm{dt}, J=15.3,6.6 \mathrm{~Hz}), 5.82-5.91(1 \mathrm{H}$, m), $6.10(1 \mathrm{H}, \mathrm{d}, J=10.2 \mathrm{~Hz}), 6.49(1 \mathrm{H}, \mathrm{d}, J=7.8 \mathrm{~Hz}), 6.80-6.84$ (2H, m), $7.00(1 \mathrm{H}, \mathrm{d}, J=7.3 \mathrm{~Hz}), 7.14-7.45(32 \mathrm{H}, \mathrm{m}) ;{ }^{13} \mathrm{C}-\mathrm{NMR}$ $\left(100 \mathrm{MHz}, \mathrm{CDCl}_{3}\right) \delta:-4.8,-4.5,11.7,13.6,13.7,17.9,22.3$, 25.8 (3C), 27.2, 27.6, 31.1, 31.2, 31.4, 32.9, 34.1, 40.0, 42.4, $52.4,53.2,55.2,56.0,65.1,66.6,67.0,69.5,70.1,76.5,113.9$ (2C), 118.2, 126.6 (3C), 126.8 (3C), 127.8 (6C), 128.0 (6C), 129.48 (6C), 129.50 (6C), 129.66, 129.73 (2C), 130.0, 132.1, 133.0, 144.3 (3C), 144.8 (3C), 159.3, 169.7, 171.2, 171.5, 171.8; HR-FAB-MS m/z: 1308.6550 (Calcd for $\mathrm{C}_{79} \mathrm{H}_{98} \mathrm{~N}_{3} \mathrm{O}_{8} \mathrm{~S}_{2} \mathrm{~S}$ $\left.[\mathrm{M}+\mathrm{H}]^{+} 1308.6565\right)$.

$(7 S, 11 R, 14 S, 17 R, 18 S, E)$-Allyl 17-[(S)-sec-Butyl]-11-butyl18-(tert-butyldimethylsilyloxy)-7-hydroxy-9,12,15-trioxo1,1,1-triphenyl-14-(tritylthiomethyl)-2-thia-10,13,16-triazaicos-5-en-20-oate (16) 2,3-Dichloro-5,6-dicyano-1,4-benzoquinone (DDQ) $(112 \mathrm{mg}, 0.50 \mathrm{mmol})$ was added in small portions to a stirred solution of $15(324 \mathrm{mg}, 0.25 \mathrm{mmol})$ in $\mathrm{CH}_{2} \mathrm{Cl}_{2}-\mathrm{H}_{2} \mathrm{O} 9: 1(12 \mathrm{~mL})$ at room temperature. After $3 \mathrm{~h}$, the mixture was diluted with $\mathrm{CHCl}_{3}(60 \mathrm{~mL})$, and the organic layer was washed with saturated aqueous $\mathrm{NaHCO}_{3}(2 \times 20 \mathrm{~mL})$ and brine $(2 \times 20 \mathrm{~mL})$, then dried over $\mathrm{MgSO}_{4}$. Concentration of the solvent in vacuo afforded a residue, which was purified by column chromatography (hexane-EtOAc, $2: 1$ ) to give $16(262 \mathrm{mg}, 89 \%)$ as a colorless viscous liquid. $[\alpha]_{\mathrm{D}}^{25}+11.6$ $\left(c=1.0, \mathrm{CHCl}_{3}\right)$; IR (neat) $\mathrm{cm}^{-1}: 3272,3060,2956,2929,2856$, $1737,1672,1634,1542,1490,1444,1387,1254,1181,1084$, 1035, 985, 938, 836, 743, 699; ${ }^{1} \mathrm{H}-\mathrm{NMR}\left(400 \mathrm{MHz}, \mathrm{CDCl}_{3}\right) \delta$ : $0.00(3 \mathrm{H}, \mathrm{s}), 0.06(3 \mathrm{H}, \mathrm{s}), 0.77-0.87(19 \mathrm{H}, \mathrm{m}), 1.06-1.12(1 \mathrm{H}$, $\mathrm{m}), 1.18-1.33(5 \mathrm{H}, \mathrm{m}), 1.48-1.57(1 \mathrm{H}, \mathrm{m}), 1.73-1.81(2 \mathrm{H}, \mathrm{m})$, 2.04-2.09 (2H, m), 2.17-2.22 (3H, m), $2.31(1 \mathrm{H}, \mathrm{dd}, J=13.7$, $2.4 \mathrm{~Hz}), 2.41(1 \mathrm{H}, \mathrm{dd}, J=16.1,6.8 \mathrm{~Hz}), 2.54-2.63(2 \mathrm{H}, \mathrm{m})$, $3.14(1 \mathrm{H}, \mathrm{d}, J=3.4 \mathrm{~Hz}), 3.88-3.98(2 \mathrm{H}, \mathrm{m}), 4.14(1 \mathrm{H}, \mathrm{td} J=7.4$, $3.4 \mathrm{~Hz}), 4.27(1 \mathrm{H}, \mathrm{td}, J=8.2,4.7 \mathrm{~Hz}), 4.32-4.36(1 \mathrm{H}, \mathrm{m}), 4.42$ $(1 \mathrm{H}, \mathrm{ddt}, J=8.4,4.5,1.9 \mathrm{~Hz}), 4.50(1 \mathrm{H}, \mathrm{ddt}, J=8.4,4.4,2.0 \mathrm{~Hz})$, 5.17-5.20 (1H, m), $5.27(1 \mathrm{H}, \mathrm{dq}, J=17.1,1.5 \mathrm{~Hz}), 5.35(1 \mathrm{H}, \mathrm{dd}$, $J=15.4,6.1 \mathrm{~Hz}), 5.48(1 \mathrm{H}, \mathrm{dt}, J=15.3,6.7 \mathrm{~Hz}), 5.79-5.89(1 \mathrm{H}$, m), $5.95(1 \mathrm{H}, \mathrm{d}, J=10.2 \mathrm{~Hz}), 6.02(1 \mathrm{H}, \mathrm{d}, J=7.8 \mathrm{~Hz}), 6.92(1 \mathrm{H}$, d, $J=8.3 \mathrm{~Hz}), 7.15-7.43(30 \mathrm{H}, \mathrm{m}) ;{ }^{13} \mathrm{C}-\mathrm{NMR}\left(100 \mathrm{MHz}, \mathrm{CDCl}_{3}\right)$ $\delta:-4.8,-4.5,11.7,13.7,13.8,18.0,22.3,25.8$ (3C), 27.1, 27.7, $31.3,31.4$ (2C), 33.1, 34.1, 40.0, 44.0, 52.5, 53.7, 56.1, 65.2, $66.6,67.0,69.4,69.7,118.3,126.6(3 \mathrm{C}), 126.8(3 \mathrm{C}), 127.8(6 \mathrm{C})$, 128.1 (6C), 129.5 (6C), 129.6 (6C), 129.9, 132.1, 132.6, 144.3 (3C), 144.9 (3C), 170.1, 171.5, 171.59, 171.61; HR-FAB-MS m/z: 1210.5809 (Calcd for $\mathrm{C}_{71} \mathrm{H}_{89} \mathrm{~N}_{3} \mathrm{O}_{7} \mathrm{~S}_{2} \mathrm{SiNa}[\mathrm{M}+\mathrm{Na}]^{+} 1210.5809$ ).

$(7 S, 11 R, 14 S, 17 R, 18 S, E)-17-[(S)$-sec-Butyl]-11-butyl-18(tert-butyldimethylsilyloxy)-7-hydroxy-9,12,15-trioxo-1,1,1triphenyl-14-(tritylthiomethyl)-2-thia-10,13,16-triazaicos-5en-20-oic Acid (9) Morpholine $(38.7 \mu \mathrm{L}, 0.44 \mathrm{mmol})$ was added dropwise to a stirred solution of $16(262 \mathrm{mg}, 0.22 \mathrm{mmol})$ in THF $(11 \mathrm{~mL})$ containing $\mathrm{Pd}\left(\mathrm{PPh}_{3}\right)_{4} \quad(25.5 \mathrm{mg}, 22 \mu \mathrm{mol})$ at room temperature under argon. After $1 \mathrm{~h}$, the reaction mixture was diluted with EtOAc $(30 \mathrm{~mL})$, and the organic layer was washed with $10 \%$ aqueous $\mathrm{HCl}(2 \times 10 \mathrm{~mL})$ and brine $(2 \times 10 \mathrm{~mL})$, then dried over $\mathrm{Na}_{2} \mathrm{SO}_{4}$. Concentration of the solvent in vacuo afforded a residue, which was purified by column chromatography $\left(\mathrm{CHCl}_{3}-\mathrm{MeOH} 40: 1\right)$ to give $9(234 \mathrm{mg}, 92 \%)$ as a colorless amorphous solid. $[\alpha]_{\mathrm{D}}^{25}+0.6$ $\left(c=1.0, \mathrm{CHCl}_{3}\right)$; IR (neat) $\mathrm{cm}^{-1}: 3284,3059,3018,2957,2929$, 2857, 1714, 1635, 1541, 1490, 1444, 1254, 1217, 1185, 1094, 1034, 951, 836, 753, 700; ${ }^{1} \mathrm{H}-\mathrm{NMR}\left(400 \mathrm{MHz}, \mathrm{CDCl}_{3}\right) \delta: 0.05$ $(6 \mathrm{H}, \mathrm{s}), 0.80(3 \mathrm{H}, \mathrm{d}, J=6.8 \mathrm{~Hz}), 0.83-0.88(15 \mathrm{H}, \mathrm{m}), 1.06-1.14$
(1H, m), 1.19-1.28 (5H, m), 1.54-1.59 (1H, m), 1.78-1.84 $(2 \mathrm{H}, \mathrm{m}), 2.03-2.08(2 \mathrm{H}, \mathrm{m}), 2.17-2.26(3 \mathrm{H}, \mathrm{m}), 2.32(1 \mathrm{H}, \mathrm{dd}$, $J=13.7,2.9 \mathrm{~Hz}), 2.39(1 \mathrm{H}, \mathrm{dd}, J=16.1,3.9 \mathrm{~Hz}), 2.45(1 \mathrm{H}, \mathrm{dd}$, $J=12.4,5.6 \mathrm{~Hz}), 2.54(1 \mathrm{H}, \mathrm{dd}, J=16.3,6.6 \mathrm{~Hz}), 2.75(1 \mathrm{H}, \mathrm{dd}$, $J=12.0,7.6 \mathrm{~Hz}), 3.92(1 \mathrm{H}, \mathrm{td}, J=13.2,4.8 \mathrm{~Hz}), 4.06-4.14(2 \mathrm{H}$, m), 4.30-4.35 (2H, m), $5.34(1 \mathrm{H}, \mathrm{dd}, J=15.4,6.1 \mathrm{~Hz}), 5.46$ $(1 \mathrm{H}, \mathrm{dt}, J=15.3,6.6 \mathrm{~Hz}), 6.36(2 \mathrm{H}, \mathrm{d}, J=9.8 \mathrm{~Hz}), 7.02(1 \mathrm{H}$, brs), 7.15-7.42 (30H, m); ${ }^{13} \mathrm{C}-\mathrm{NMR}\left(100 \mathrm{MHz}, \mathrm{CDCl}_{3}\right) \delta:-4.9$, $-4.3,11.8,13.4,13.8,17.9,22.3,25.7$ (3C), 27.2, 27.8, 29.7, $31.3,31.4,33.1,34.2,40.3,44.1,53.1,53.8,57.0,66.6,67.0$, 68.9, 69.7, 126.6 (3C), 126.9 (3C), 127.9 (6C), 128.1 (6C), 129.5 (6C), 129.6 (6C), 130.0, 132.3, 144.3 (3C), 144.9 (3C), 170.3, 171.9 (2C), 172.3; HR-FAB-MS $m / z$ : 1170.5470 (Calcd for $\left.\mathrm{C}_{68} \mathrm{H}_{85} \mathrm{~N}_{3} \mathrm{O}_{7} \mathrm{~S}_{2} \mathrm{SiNa}[\mathrm{M}+\mathrm{Na}]^{+} 1170.5496\right)$.

$(2 S, 6 R, 9 S, 12 R, 13 S)-12-[(S)$-sec-Butyl]-6-butyl-13-(tertbutyldimethylsilyloxy)-2-[(E)-4-(tritylthio)but-1-en-1-yl]9-tritylthiomethyl-1-oxa-5,8,11-triazacyclopentadecane4,7,10,15-tetraone (17) A solution of $9(191 \mathrm{mg}, 0.17 \mathrm{mmol})$ in $\mathrm{CH}_{2} \mathrm{Cl}_{2}(17 \mathrm{~mL})$ was added very slowly to a stirred solution of MNBA (74.4 mg, $0.22 \mathrm{mmol})$ in $\mathrm{CH}_{2} \mathrm{Cl}_{2}(170 \mathrm{~mL}, 1.0 \mathrm{~mm}$ concentration) containing DMAP $(61.0 \mathrm{mg}, 0.50 \mathrm{mmol})$ at toom temperature over $14 \mathrm{~h}$. After $1 \mathrm{~h}$, the mixture was diluted with $\mathrm{CH}_{2} \mathrm{Cl}_{2}(200 \mathrm{~mL})$, and the organic layer was washed successively with saturated aqueous $\mathrm{NaHCO}_{3}(2 \times 80 \mathrm{~mL})$, water $(2 \times 80 \mathrm{~mL})$ and brine $(2 \times 80 \mathrm{~mL})$, then dried over $\mathrm{MgSO}_{4}$. Concentration of the solvent in vacuo afforded a residue, which was purified by column chromatography (hexane-EtOAc, $2: 1$ ) to give $17(160 \mathrm{mg}, 85 \%)$ as a colorless amorphous solid. $[\alpha]_{\mathrm{D}}^{25}$ $-6.4\left(c=1.0, \mathrm{CHCl}_{3}\right)$; IR (neat) $\mathrm{cm}^{-1}: 3286,3059,3031,2956$, 2928, 2857, 1733, 1652, 1539, 1490, 1444, 1258, 1198, 1098, 1034, 990, 834, 743, $700 \mathrm{~cm}^{-1} ;{ }^{1} \mathrm{H}-\mathrm{NMR}\left(400 \mathrm{MHz}, \mathrm{CDCl}_{3}\right) \delta$ : $-0.10(3 \mathrm{H}, \mathrm{s}), 0.00(3 \mathrm{H}, \mathrm{s}), 0.76(3 \mathrm{H}, \mathrm{d}, J=6.8 \mathrm{~Hz}), 0.79-0.86$ $(15 \mathrm{H}, \mathrm{m}), 0.98-1.05(1 \mathrm{H}, \mathrm{m}), 1.12-1.19(1 \mathrm{H}, \mathrm{m}), 1.25-1.38(4 \mathrm{H}$, m), 1.58-1.66 (1H, m), 1.75-1.86 (2H, m), 2.00-2.07 (2H, m), $2.16-2.20(2 \mathrm{H}, \mathrm{m}), 2.30(1 \mathrm{H}, \mathrm{dd}, J=16.6,5.9 \mathrm{~Hz}), 2.35-2.43$ $(2 \mathrm{H}, \mathrm{m}), 2.48(1 \mathrm{H}, \mathrm{dd}, J=13.9,3.2 \mathrm{~Hz}), 2.55-2.62(1 \mathrm{H}, \mathrm{m})$, $3.24-3.28(2 \mathrm{H}, \mathrm{m}), 3.70(1 \mathrm{H}, \mathrm{td}, J=10.2,2.0 \mathrm{~Hz}), 3.99-4.04$ $(1 \mathrm{H}, \mathrm{m}), 4.10-4.15(1 \mathrm{H}, \mathrm{m}), 5.34(1 \mathrm{H}, \mathrm{dd}, J=15.6,7.3 \mathrm{~Hz})$, 5.51-5.56 (1H, m), $5.66(1 \mathrm{H}, \mathrm{dt}, J=15.3,6.7 \mathrm{~Hz}), 6.13(1 \mathrm{H}, \mathrm{d}$, $J=7.8 \mathrm{~Hz}), 6.82(1 \mathrm{H}, \mathrm{d}, J=5.9 \mathrm{~Hz}), 7.19-7.43 \mathrm{ppm}(31 \mathrm{H}, \mathrm{m})$; ${ }^{13} \mathrm{C}-\mathrm{NMR}\left(100 \mathrm{MHz}, \mathrm{CDCl}_{3}\right) \delta:-4.9,-3.9,12.0,13.0,13.8$, $17.9,22.4,25.8$ (3C), 27.4, 27.9, 30.9, 31.0, 31.3, 31.9, 34.0, $41.8,41.9,54.0,56.6,58.1,66.6,66.8,68.7,71.8,126.6(3 \mathrm{C})$, 126.8 (3C), 127.9 (6C), 128.0 (6C), 128.1, 129.48 (6C), 129.53 (6C), 133.4, 144.4 (3C), 144.8 (3C), 169.5, 170.2, 170.4, 173.0; HR-FAB-MS $m / z$ : 1152.5363 (Calcd for $\mathrm{C}_{68} \mathrm{H}_{83} \mathrm{~N}_{3} \mathrm{O}_{6} \mathrm{~S}_{2} \mathrm{SiNa}$ $\left.[\mathrm{M}+\mathrm{Na}]^{+} 1152.5390\right)$.

$(1 S, 5 S, 6 R, 9 S, 20 R, E)-6-[(S)-s e c-B$ utyl]-20-butyl5-(tert-butyldimethylsilyloxy)-2-oxa-11,12-dithia-7,19,22triazabicyclo[7.7.6]docos-15-ene-3,8,18,21-tetraone (18) A solution of $17(156 \mathrm{mg}, 0.14 \mathrm{mmol})$ in $\mathrm{CH}_{2} \mathrm{Cl}_{2}-\mathrm{MeOH} 9: 1$ $(35 \mathrm{~mL})$ was added dropwise to a vigorously stirred solution of $\mathrm{I}_{2}(350 \mathrm{mg}, 1.4 \mathrm{mmol})$ in $\mathrm{CH}_{2} \mathrm{Cl}_{2}-\mathrm{MeOH} 9: 1(280 \mathrm{~mL}, 0.5 \mathrm{~mm}$ concentration) over $10 \mathrm{~min}$ at room temperature. After $10 \mathrm{~min}$, the reaction was quenched with $10 \%$ aqueous $\mathrm{Na}_{2} \mathrm{~S}_{2} \mathrm{O}_{3}(60 \mathrm{~mL})$ at room temperature. The resulting mixture was diluted with $\mathrm{CH}_{2} \mathrm{Cl}_{2}(150 \mathrm{~mL})$, and the organic layer was washed with saturated aqueous $\mathrm{NaHCO}_{3}(2 \times 60 \mathrm{~mL})$ and brine $(2 \times 60 \mathrm{~mL})$, then dried over $\mathrm{MgSO}_{4}$. Concentration of the solvent in vacuo afforded a residue, which was purified by column chromatography $\left(\mathrm{CHCl}_{3}-\mathrm{MeOH} 40: 1\right)$ to give $18(84.2 \mathrm{mg}, 95 \%)$ as a 
colorless amorphous solid. $[\alpha]_{\mathrm{D}}^{25}+5.7\left(c=1.0, \mathrm{CHCl}_{3}\right)$; IR (neat) $\mathrm{cm}^{-1}:$ 3339, 2957, 2930, 2858, 1747, 1661, 1542, 1463, 1433, 1362, 1257, 1158, 1138, 1080, 1027, 989, 949, 837, 810, 775, $755 \mathrm{~cm}^{-1} ;{ }^{1} \mathrm{H}-\mathrm{NMR}\left(400 \mathrm{MHz}, \mathrm{CDCl}_{3}\right) \delta: 0.09(3 \mathrm{H}, \mathrm{s}), 0.16$ $(3 \mathrm{H}, \mathrm{s}), 0.88(3 \mathrm{H}, \mathrm{d}, J=6.8 \mathrm{~Hz}), 0.90-0.95(15 \mathrm{H}, \mathrm{m}), 1.21-1.28$ $(1 \mathrm{H}, \mathrm{m}), 1.35-1.49(5 \mathrm{H}, \mathrm{m}), 1.64-1.72(1 \mathrm{H}, \mathrm{m}), 1.85-1.98(2 \mathrm{H}$, $\mathrm{m}), 2.58-2.62(3 \mathrm{H}, \mathrm{m}), 2.73-2.76(3 \mathrm{H}, \mathrm{m}), 2.92-3.13(4 \mathrm{H}$, m), $3.68(1 \mathrm{H}$, brs $), 4.20-4.24(1 \mathrm{H}, \mathrm{m}), 4.86(1 \mathrm{H}, \mathrm{dt}, J=10.2$, $3.4 \mathrm{~Hz}), 5.00-5.05(1 \mathrm{H}, \mathrm{m}), 5.65(1 \mathrm{H}, \mathrm{d}, J=15.6 \mathrm{~Hz}), 5.70-5.72$ $(1 \mathrm{H}, \mathrm{m}), 5.81(1 \mathrm{H}, d, J=3.9 \mathrm{~Hz}), 6.48(1 \mathrm{H}, \mathrm{brs}), 6.69(1 \mathrm{H}, \mathrm{d}$, $J=9.8 \mathrm{~Hz}), 7.09 \mathrm{ppm}(1 \mathrm{H}, \mathrm{d}, J=6.8 \mathrm{~Hz}) ;{ }^{13} \mathrm{C}-\mathrm{NMR}(100 \mathrm{MHz}$, $\left.\mathrm{CDCl}_{3}\right) \delta:-4.9,-4.3,11.9,13.8,14.5,17.9,22.2,25.7$ (3C), $27.8,28.5,30.8,34.9,36.3,39.6,40.5,41.2,43.5,53.4,56.4$, 61.1, 67.1, 68.7, 128.7, 133.0, 169.0 (2C), 170.5, 170.9; HRFAB-MS $m / z$ : 644.3228 (Calcd for $\mathrm{C}_{30} \mathrm{H}_{54} \mathrm{~N}_{3} \mathrm{O}_{6} \mathrm{~S}_{2} \mathrm{Si}[\mathrm{M}+\mathrm{H}]^{+}$ 644.3223).

Thailandepsin B (2) $\mathrm{HF} \cdot$ pyridine $(1.3 \mathrm{~mL})$ was added to a stirred solution of $\mathbf{1 8}(83.2 \mathrm{mg}, 0.13 \mathrm{mmol})$ in pyridine $(2.6 \mathrm{~mL})$ at room temperature. After $22 \mathrm{~h}$, the reaction mixture was diluted with EtOAc $(60 \mathrm{~mL})$, and the organic layer was washed successively with $3 \%$ aqueous $\mathrm{HCl}(3 \times 15 \mathrm{~mL})$, saturated aqueous $\mathrm{NaHCO}_{3}(2 \times 15 \mathrm{~mL})$ and brine $(2 \times 15 \mathrm{~mL})$, then dried over $\mathrm{Na}_{2} \mathrm{SO}_{4}$. Concentration of the solvent in vacuo afforded a residue, which was purified by column chromatography $\left(\mathrm{CHCl}_{3}-\right.$ $\mathrm{MeOH} 10: 1)$ to give 2 (thailandepsin B) $(61.2 \mathrm{mg}, 90 \%)$ as a white amorphous solid. $[\alpha]_{\mathrm{D}}^{25}-28.4(c=1.0, \mathrm{MeCN}),\left\{\right.$ lit. ${ }^{1)}$ $\left.[\alpha]_{\mathrm{D}}^{25}-22.8(c=1.0, \mathrm{MeCN})\right\}$. The IR, ${ }^{1} \mathrm{H}-$ and ${ }^{13} \mathrm{C}-\mathrm{NMR}$, and MS spectra were identical to those of natural thailandepsin $\mathrm{B}^{1)}$; IR (neat) $\mathrm{cm}^{-1}: 3330,2960,2931,2873,1733,1660,1540$, 1457, 1434, 1405, 1375, 1277, 1162, 1049, 1023, 981, 910, 731; ${ }^{1} \mathrm{H}-\mathrm{NMR}\left(600 \mathrm{MHz}, \mathrm{CDCl}_{3}\right) \quad \delta: 0.91-0.95(9 \mathrm{H}, \mathrm{m}), 1.21-1.28$ $(1 \mathrm{H}, \mathrm{m}), 1.36-1.49(4 \mathrm{H}, \mathrm{m}), 1.51-1.56(1 \mathrm{H}, \mathrm{m}), 1.60-1.74(1 \mathrm{H}$, m), 1.91-1.97 (1H, m), 2.08-2.12 (1H, m), 2.46-2.51 (1H, m), $2.59(1 \mathrm{H}, \mathrm{dd}, J=13.2,1.1 \mathrm{~Hz}), 2.70-2.75(4 \mathrm{H}, \mathrm{m}), 2.86(1 \mathrm{H}, \mathrm{d}$, $J=10.6 \mathrm{~Hz}), 2.91-2.95(1 \mathrm{H}, \mathrm{m}), 3.08-3.12(1 \mathrm{H}, \mathrm{m}), 3.15-3.22$ (1H, m), $3.36(1 \mathrm{H}, \mathrm{dd}, J=13.4,7.1 \mathrm{~Hz}), 3.41-3.49(1 \mathrm{H}, \mathrm{m})$, 4.17-4.20 (1H, m), 4.63-4.67 (1H, m), $4.94(1 \mathrm{H}, \mathrm{td}, J=8.6$, $3.3 \mathrm{~Hz}), 5.50-5.51(1 \mathrm{H}, \mathrm{m}), 5.67(1 \mathrm{H}, \mathrm{d}, J=15.4 \mathrm{~Hz}), 5.90(1 \mathrm{H}$, d, $J=3.7 \mathrm{~Hz}), 6.47(1 \mathrm{H}$, br s), $6.72(1 \mathrm{H}, \mathrm{d}, J=9.5 \mathrm{~Hz}), 7.25(1 \mathrm{H}$, d, $J=7.0 \mathrm{~Hz}) ;{ }^{13} \mathrm{C}-\mathrm{NMR}\left(150 \mathrm{MHz}, \mathrm{CDCl}_{3}\right) \delta: 11.6,13.8,15.3$, $22.2,27.1,28.4,30.6,33.7,36.3,39.5,40.5,40.8,41.9,54.2$, 56.6, 61.9, 68.3, 70.4, 128.3, 133.7, 169.1, 170.5, 170.7, 171.9; HR-FAB-MS m/z: 530.2360 (Calcd for $\mathrm{C}_{24} \mathrm{H}_{40} \mathrm{~N}_{3} \mathrm{O}_{6} \mathrm{~S}_{2}[\mathrm{M}+\mathrm{H}]^{+}$ 530.2359).

Acknowledgments This study was supported by a Grantin-Aid for the Strategic Research Foundation Program at Private Universities (No. S1511001L, 2015-2019) and a Grant-
in-Aid for Scientific Research (C) (No. 15K07865, 2015-2017) from the Ministry of Education, Culture, Sports, Science and Technology (MEXT) of Japan.

Conflict of Interest The authors declare no conflict of interest.

Supplementary Materials The online version of this article contains supplementary materials.

\section{References and Notes}

1) Wang C., Henkes L. M., Doughty L. B., He M., Wang D., MeyerAlmes F.-J., Cheng Y.-Q., J. Nat. Prod., 74, 2031-2038 (2011).

2) Biggins J. B., Gleber C. D., Brady S. F., Org. Lett., 13, 1536-1539 (2011).

3) VanderMolen K. M., McCulloch W., Pearce C. J., Oberlies N. H., J. Antibiot., 64, 525-531 (2011).

4) Grant C., Rahman F., Piekarz R., Peer C., Frye R., Robey R. W., Gardner E. R., Figg W. D., Bates S. E., Expert Rev. Anticancer Ther., 10, 997-1008 (2010).

5) Shah M. H., Binkley P., Chan K., Xiao J., Arbogast D., Collamore M., Farra Y., Young D., Grever M., Clin. Cancer Res., 12, $3997-$ 4003 (2006).

6) Benelkerbir H., Donlevy A. M., Packham G., Ganesan A., Org. Lett., 13, 6337-6344 (2011).

7) Fukui Y., Narita K., Dan S., Yamori T., Ito A., Yoshida M., Katoh T., Eur. J. Med. Chem., 76, 301-313 (2014).

8) Cheng Y.-Q., Yang S., Wang P. (ChinAm PharmaTech Wuhan Co., Ltd., China), PCT WO 131355 A1 (2015).

9) Narita K., Kikuchi T., Watanabe K., Takizawa T., Oguchi T., Kudo K., Matsuhara K., Abe H., Yamori T., Yoshida M., Katoh T., Chem. Eur. J., 15, 11174-11186 (2009).

10) Takizawa T., Watanabe K., Narita K., Kudo K., Oguchi T., Abe H., Katoh T., Heterocycles, 76, 275-290 (2008).

11) Takizawa T., Watanabe K., Narita K., Oguchi T., Abe H., Katoh T., Chem. Commun., 1677-1679 (2008).

12) Narita K., Fukui Y., Sano Y., Yamori T., Ito A., Yoshida M., Katoh T., Eur. J. Med. Chem., 60, 295-304 (2013).

13) Shiina I., Katoh T., Nagai S., Hashizume M., Chem. Rec., 9, 305320 (2009).

14) Shiina I., Sasaki A., Kikuchi T., Fukui H., Chem. Asian J., 3, 462472 (2008).

15) Shiina I., Chem. Rev., 107, 239-273 (2007).

16) Shiina I., Hashizume M., Tetrahedron, 62, 7934-7939 (2006).

17) Shiina I., Kubota M., Oshiumi H., Hashizume M., J. Org. Chem., 69, 1822-1830 (2004).

18) In this condensation reaction, we used 1.5 equiv. of 12 to smoothly complete the reaction. This is because the condensation partner $\mathbf{1 3}$ showed low reactivity to $\mathbf{1 2}$. In addition, compound 13, prepared from commercially available material via a ten-step sequence, was more valuable than compound $\mathbf{1 2}$. 